\section{La regulación de la protección radiológica y la función de las autoridades de salud}

\author{
César F. Arias ${ }^{1}$
}

Forma de citar: Arias CF. La regulación de la protección radiológica y la función de las autoridades de salud. Rev Panam Salud Publica. 2006;20(2/3);188-97.

Palabras clave: radiación ionizante, protección radiológica, control de la radiación, reglamentos.

\footnotetext{
1 Departamento de Física, Universidad de Buenos Aires, Paseo Colón 850, CP1063, Buenos Aires, Argentina. Correo electrónico: carias@ fi.uba.ar
}

\begin{abstract}
SINOPSIS
En este artículo se presenta una breve sintesis de la evolución de la protección contra las radiaciones ionizantes y se hace una interpretación de su filosofía actual. Se analiza el papel decisivo que deben desempeñar las organizaciones reguladoras en protección radiológica y la importante contribución que pueden brindar las autoridades sanitarias. Estas deberian participar activamente al menos en tres aspectos: la promoción de la educación formal del personal de salud en lo concerniente a la protección radiológica, la atención médica de las personas sobreexpuestas accidentalmente y la protección radiológica de los pacientes en relación con los procedimientos radiológicos. Para lograr esos objetivos, los profesionales sanitarios han de tener los conocimientos necesarios en materia de protección radiológica, promover el uso de los equipos adecuados y aplicar los procedimientos necesarios de garantía de la calidad. La apropiada intervención de las autoridades nacionales de salud puede contribuir en gran medida a reducir las dosis innecesarias en los procedimientos médicos con fuentes de radiación y reducir la probabilidad de que ocurran accidentes radiológicos en este campo.
\end{abstract}

La posibilidad de obtener imágenes del interior de la materia sorprendió al mundo cuando Wilhelm Conrad Roentgen descubrió los rayos X en la Universidad de Würzburgo, Alemania, en 1895. Un año después, Antoine Henri Becquerel comenzó a explorar otro fenómeno que Marie Curie denominó más tarde "radiactividad". Todos ellos fueron acreedores del Premio Nóbel de Física en reconocimiento a descubrimientos que abrían un amplio campo al conocimiento y a la imaginación.

En la actualidad, innumerables aplicaciones derivadas de aquellos primeros conocimientos son práctica habitual en diversas áreas de la producción, la investigación y - de manera muy especial- la medicina. Pocos descubrimientos han tenido un impacto tan grande en el campo médico. La radiología convencional, la tomografía computarizada, la radiología intervencionista, las técnicas de medicina nuclear, la tomografía por emisión de positrones y la radioterapia con fuentes radiactivas y aceleradores de partículas son procedimientos frecuentes en la medicina moderna.

Sin embargo, casi a raíz de su descubrimiento se hizo evidente que los rayos $X$ y la radiactividad también podían causar daños a la salud. Ya en 1896 se observaron problemas de depilación, eritemas, quemaduras, amputaciones —e incluso la muerteen las personas que empleaban tubos de rayos 
$\mathrm{X}$ y materiales radiactivos en sus investigaciones. A medida que los pioneros de la radiología sufrían afecciones o morían prematuramente, los científicos comprendieron una contradictoria realidad: estos nuevos descubrimientos que podían contribuir a salvar la vida también podían destruirla.

En este artículo se presenta una breve síntesis de la evolución de las medidas de protección contra las radiaciones ionizantes y se hace una interpretación de la filosofía actual al respecto. Además, se analizan la importancia de contar con organizaciones reguladoras que sean eficaces en este sentido y la significativa contribución que pueden aportar las autoridades de salud, especialmente en la protección de pacientes sometidos a procedimientos médicos de diagnóstico o a tratamientos en que se emplean fuentes de radiación ionizante.

\section{ORIGEN Y EVOLUCIÓN DE LA PROTECCIÓN RADIOLÓGICA}

En la figura 1 se resume la evolución de los límites de dosis, concepto central de la protección radiológica, es decir, de la disciplina dedicada a la protección de las personas contra los peligros de las radiaciones. Sin embargo, para interpretar correctamente esta figura es necesario comprender cómo ha progresado el conocimiento en radiobiología, el significado de las magnitudes y unidades de medida adoptadas para correlacionar la exposición a las radiaciones con sus riesgos y los criterios adoptados a lo largo del tiempo en torno a la protección de las personas $(1,2)$.

FIGURA 1. Límites de exposición anual recomendados para los trabajadores por la Comisión Internacional de Protección Radiológica, 1934-1990ª

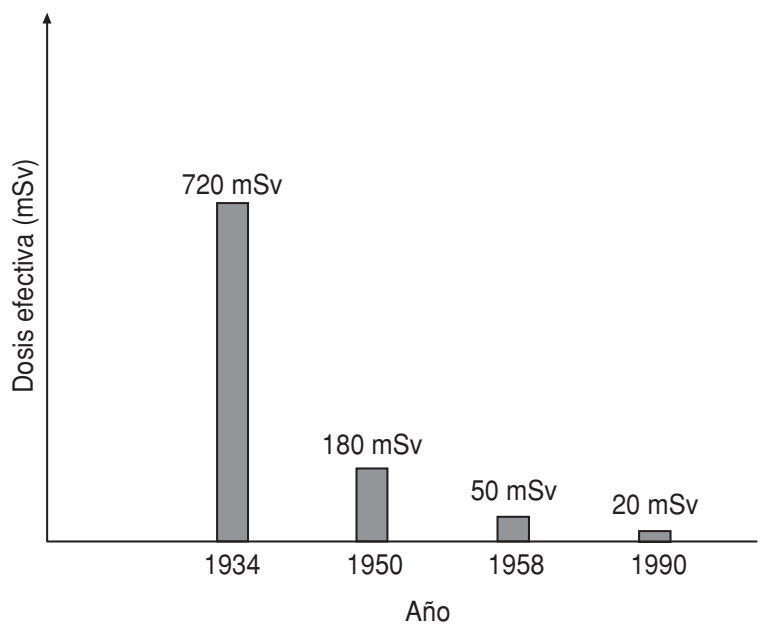

Fuente: Datos tomados de las referencias 1 y 2

a En la actualidad está vigente el límite establecido en 1990.
Los rayos $X$, las emisiones radiactivas (alfa, beta y gamma, entre otras) y las partículas resultantes de reacciones nucleares (como los neutrones, los protones y los deuterones) pueden afectar a la salud de las personas - aun cuando la energía absorbida sea muy pequeña - debido a la capacidad que poseen estas radiaciones de ionizar los átomos que encuentran en su trayecto, peculiaridad que les valió la denominación de radiaciones ionizantes. Las moléculas que poseen átomos ionizados aumentan su reactividad química y pueden provocar alteraciones en las estructuras celulares, siendo de particular importancia las que ocurren en las moléculas de ácido desoxirribonucleico (ADN). Otras radiaciones electromagnéticas de mayor longitud de onda $-\mathrm{y}$ por consiguiente de menor energía fotónica-, denominadas radiaciones no ionizantes, no tienen esa posibilidad y solo pueden afectar a la salud mediante otros procesos biofísicos que requieren niveles de exposición miles de veces superiores en términos de la energía absorbida (3).

\section{Los efectos de las radiaciones ionizantes y el inicio de la protección radiológica}

Los médicos fueron los primeros en emplear fuentes de radiaciones ionizantes para realizar exploraciones radiológicas y también en sufrir los daños provocados por las radiaciones. Esto llevó a que en el Segundo Congreso Internacional de Radiología, celebrado en Estocolmo, Suecia, en 1928, se recomendara la creación de un organismo internacional para ocuparse de este problema. Así nació la protección radiológica como disciplina y se creó un organismo que en la actualidad se denomina Comisión Internacional de Protección Radiológica (CIPR).

Durante el Primer Congreso Internacional de Radiología, celebrado en Londres, Reino Unido, en 1925, se había creado la Comisión Internacional de Unidades y Medidas de Radiación (CIUR) con el fin de proponer magnitudes y unidades de medida apropiadas para evaluar la exposición a las radiaciones. Tanto la CIUR como la CIPR —dos organizaciones nacidas de la radiología médica- han contribuido a definir las magnitudes y unidades que se emplean en la protección radiológica (cuadro 1).

Algunos experimentos llevados a cabo en animales e investigaciones realizadas con personas expuestas a radiaciones ionizantes por razones médicas o laborales han contribuido a conocer los efectos biológicos de dichas radiaciones. Sin embargo, la mayor investigación epidemiológica llevada a cabo hasta la fecha es la realizada en las poblaciones japonesas de Hiroshima y Nagasaki con la participación de los sobrevivientes de las explosiones atómicas de agosto de 1945. En ese estudio, que aún no ha concluido, han participado alrededor de 86000 per- 
CUADRO 1. Magnitudes y unidades empleadas en la protección radiológica

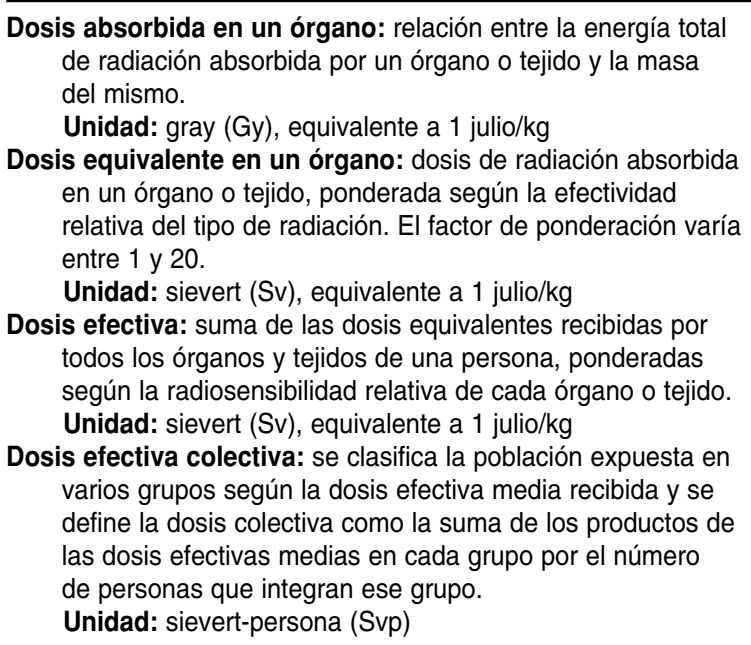

Fuente: Datos tomados de las referencias 1 y 4.

sonas. Además, como consecuencia del accidente nuclear que ocurrió en 1986 en Chernobyl, antigua Unión Soviética, se ha reunido información importante sobre la incidencia de cáncer de tiroides en niños y niñas expuestos a las radiaciones.

En 1955, la Organización de las Naciones Unidas creó el Comité Científico para el Estudio de los Efectos de las Radiaciones Atómicas (ONU/CCEERA) con el fin de recopilar información sobre ese particular. Desde entonces, este comité publica periódicamente informes sobre las fuentes de radiación existentes en el mundo, los niveles de exposición de las personas y los resultados de las investigaciones sobre los efectos de la radiación en la salud (5).

\section{¿Se pueden evitar del todo los efectos de las radiaciones ionizantes?}

Esta pregunta refleja la esencia del problema atañente a la filosofía de la protección radiológica. Ciertos efectos denominados "deterministas", tales como la esterilidad, la catarata, el eritema, los trastornos hematopoyéticos y el síndrome agudo por radiación, pueden evitarse del todo si las dosis que reciben las personas no sobrepasan determinados umbrales; estos son de alrededor de 0,5 gray (Gy) en el caso de la exposición aguda y de 0,1 Gy en el de la exposición crónica. Sin embargo, otros efectos llamados "estocásticos" (la inducción del cáncer y algunos trastornos hereditarios) no pueden evitarse por completo. No hay datos comprobatorios que permitan establecer una dosis umbral para la aparición de estos efectos y se considera que cualquier exposición a las radiaciones ionizantes, por pe- queña que sea la dosis, contribuye a aumentar la probabilidad de inducción de cáncer y, si la exposición es de las gónadas, también de trastornos hereditarios. Los términos "determinista" y "estocástico" aluden a la naturaleza pronosticable o probabilística de estos efectos.

Según estimaciones de la CIPR, los trabajadores que se exponen a dosis pequeñas y a tasas de dosis de radiación bajas en su lugar de trabajo tienen una probabilidad de $4 \%$ de morir de un cáncer radioinducido por cada sievert $(\mathrm{Sv})$ de dosis efectiva recibida (1). En el caso de miembros de la población en general, que en algunas circunstancias pudieran verse expuestos a dosis pequeñas $\mathrm{y}$ a tasas de dosis de radiación bajas, la probabilidad de morir de un cáncer inducido por las radiaciones es de $5 \%$ por cada Sv de dosis efectiva recibida. Estas cifras se conocen como coeficientes de riesgo y la diferencia entre los valores correspondientes a los trabajadores y a personas de la población en general se debe a que en la categoría de los "trabajadores" no se incluye a los menores de 18 años de edad, cuya sensibilidad a las radiaciones es mayor. Estas cifras podrían verse ligeramente modificadas en las nuevas recomendaciones generales de la CIPR, no solamente debido a la actualización de la información epidemiológica, sino a una posible revisión del criterio empleado para definir los coeficientes de riesgo.

Hasta el momento no se han comprobado efectos hereditarios en la descendencia de las personas expuestas a las radiaciones; sin embargo, estudios realizados en animales permiten suponer que esos efectos estocásticos pueden ocurrir también en los seres humanos. Se estima que la relación entre los efectos hereditarios observados en la primera generación y la dosis de radiación es 10 veces menor que la relación entre los efectos cancerígenos y la dosis (6).

Por lo tanto, los efectos estocásticos de las radiaciones no se pueden evitar por completo si la exposición no es nula. A los fines de la protección radiológica se acepta la hipótesis de que la probabilidad de que ocurran estos efectos aumenta en proporción con las dosis cuando estas y las tasas de dosis son pequeñas.

\section{LA PROTECCIÓN RADIOLÓGICA EN LA ACTUALIDAD}

Las últimas recomendaciones generales elaboradas por la CIPR sobre la protección radiológica datan de 1990 (1) y se ha anunciado su actualización para fines de 2006 o principios de 2007. Aunque no se esperan cambios sustanciales en cuanto a la filosofía de la protección radiológica, es posible que en las nuevas recomendaciones generales se modifique el énfasis que antes se ponía en algunos conceptos. 


\section{Las bases filosóficas de la protección radiológica}

Los riesgos asociados con la exposición a las radiaciones dependen de las dosis de radiación que reciben las personas expuestas. Por lo tanto, para reducir esos riesgos se deben reducir las dosis que se reciben y la exposición innecesaria a las radiaciones.

La protección de las personas contra los diversos riesgos originados en el medio ambiente laboral o público siempre se ha basado en el establecimiento de límites a la presencia de sustancias contaminantes o a la exposición individual. Si bien en sus inicios la protección contra las radiaciones ionizantes se rigió por ese criterio, a partir de la década de 1970 el concepto de límite comenzó a concebirse como una referencia de riesgo máximo, tolerable solo en situaciones excepcionales. El protagonismo en la filosofía de la protección radiológica se desplazó entonces gradualmente hacia la justificación de las prácticas basadas en el uso de fuentes de radiación y la optimización de la protección radiológica.

Para un organismo internacional no es una tarea sencilla recomendar valores límites de riesgo. El conocimiento actual sobre los efectos de las radiaciones ionizantes en los seres humanos se aplica a diversas culturas y nacionalidades pero, a pesar del llamado proceso de globalización, la situación económica y social de los diversos países es extremadamente desigual. Si los límites recomendados fueran muy bajos, muchos países no podrían adoptarlos debido al alto costo que implicaría la protección, mientras que la recomendación de límites elevados no contribuiría a disminuir los riesgos en grado significativo. En ambos casos, las recomendaciones resultarían desacertadas. Ante este dilema, la CIPR decidió recomendar límites de riesgo intermedios y aplicar principios de justificación y optimización.

Dado que toda exposición a las radiaciones implica cierto riesgo, la aceptación del uso de las fuentes de radiaciones debe verse justificada por los beneficios que aporta a toda la sociedad o a una parte de ella. Por ejemplo, en la década de 1940, algunas zapaterías empleaban equipos de fluoroscopia con rayos $\mathrm{X}$ para determinar el tipo de calzado adecuado para cada cliente y hasta hace unos 15 años se montaban fuentes radiactivas en los extremos de algunos pararrayos, a pesar de que nunca se logró demostrar que esto aumentara su eficacia durante las tormentas eléctricas. En la actualidad, tales aplicaciones de las fuentes de radiación no se consideran justificadas y no se autorizan.

Optimizar la protección significa comprender que el uso de las fuentes de radiación conduce a la exposición inevitable de algunas personas que estarán tanto mejor protegidas cuanto menor sean las dosis de radiaciones que reciben, y actuar en consecuencia. Pero, ¿hasta dónde se deben reducir las dosis? La respuesta no la pueden proporcionar solamente los estudios científicos; también es necesario tener en cuenta las condiciones económicas y sociales imperantes en cada país.

No les corresponde a los organismos internacionales determinar el grado de esfuerzo económico que cada país debe realizar para proteger a sus ciudadanos contra un riesgo laboral o ambiental. Por consiguiente, el concepto de optimización es un criterio genérico - sin alusiones a valores específicos-que ha pasado a tener mayor trascendencia que los límites numéricos. Según la CIPR, se deben reducir las dosis individuales de radiación, el número de personas expuestas y la probabilidad de que ocurran exposiciones accidentales tanto como sea razonablemente posible (por debajo de los límites) teniendo en cuenta los factores económicos y sociales, es decir, las restricciones económicas y las necesidades de la sociedad (1). De este modo, los organismos internacionales compatibilizan su responsabilidad de ofrecer la mejor recomendación con la ineludible realidad de que lo mejor no es igual para todos. Queda a criterio de cada país definir sus objetivos en materia de protección radiológica tomando los límites recomendados como una cota superior. Solo en casos excepcionales, una persona quedaría expuesta a riesgos cercanos a los límites.

Cuando una fuente de radiación funciona en condiciones normales -es decir, cuando la exposición de las personas ocurre según lo planificado- se puede controlar el riesgo radiológico mediante la aplicación de los límites de dosis y las restricciones derivadas de los procesos de optimización. Los límites de dosis aplicables a los trabajadores y a los miembros del público son distintos, debido a que la relación riesgo-beneficio es diferente en cada uno de estos grupos. Las dosis que las personas reciben debido a la exposición a las radiaciones de carácter natural y las que reciben durante los procedimientos radiológicos con propósitos médicos no se deben contabilizar a efectos de la aplicación de los límites.

En el cuadro 2 se resumen los valores de los límites anuales de dosis recomendados por la CIPR en 1991. Estos límites no establecen una frontera entre el riesgo y la seguridad, sino que indican los valores de riesgo máximos tolerables recomendados por la CIPR. En el mismo cuadro se indican los valores de la probabilidad de morir de un cáncer inducido por la radiación, correspondientes a los límites de dosis, según los coeficientes de riesgo indicados por esa entidad (1). Muchos países han adoptado estos valores límite y se espera que se ratifiquen en la próxima edición de las recomendaciones de la CIPR.

Otro objetivo de la protección radiológica es reducir los riesgos asociados con los accidentes radiológicos. La CIPR ha introducido la expresión 


\section{CUADRO 2. Límites anuales de dosis efectiva recomendados por la Comisión Internacional de Pro- tección Radiológica y riesgos asociados}

\begin{tabular}{lcc}
\hline \multicolumn{1}{c}{ Grupo poblacional } & Límite de dosis efectiva & Riesgo anual de morir \\
\hline Trabajadores & $20 \mathrm{mSv}^{\mathrm{b}}$ & 8 por cada 10000 trabajadores \\
Miembros de la población & $1 \mathrm{mSv}$ & 5 por cada 100000 personas \\
\hline Fuente: Datos tomados de la referencia 1. & \\
a Promedio de las dosis efectivas recibidas en 5 años. \\
b Riesgo anual de morir de un cáncer inducido por radiaciones si se recibe una dosis anual de radiación igual al límite de dosis efectiva.
\end{tabular}

"exposición potencial" para aludir a la exposición originada durante situaciones accidentales hipotéticas y a partir de 1990 ha prestado una atención especial a la prevención de los accidentes con fuentes de radiación $(7,8)$. Con tal propósito recomienda el criterio de reducir la probabilidad de que ocurran accidentes mediante sistemas de seguridad apropiados, de modo que los riesgos radiológicos derivados de situaciones accidentales en que pueda estar involucrada una fuente de radiación sean del mismo orden de magnitud que los riesgos asociados con la exposición a las radiaciones en condiciones de operación normal de dicha fuente (8).

A pesar de los reparos formulados por la CIPR acerca de la naturaleza colectiva del criterio de optimización durante el proceso de revisión de las actuales recomendaciones (9), no cabe duda de que la aplicación conjunta y sistemática de los criterios de limitación de dosis y optimización ha constituido una estrategia eficaz para reducir los riesgos asociados con las radiaciones. Según las estadísticas compiladas por el ONU/CCEERA a partir de la información brindada por las autoridades nacionales, se observa que los valores de dosis de radiación recibidas por los trabajadores muestran una tendencia decreciente a lo largo de las últimas décadas, al menos en los países que cuentan con estructuras reguladoras apropiadas. Esos datos indican que la dosis efectiva promedio recibida anualmente por los trabajadores de distintos países se ha reducido $33 \%$ en 30 años (5). Sin embargo, se debe tener en cuenta que no todos los países cuentan con mecanismos para documentar las dosis ocupacionales y, por lo tanto, en tales casos no se tiene información sobre la evolución de las mismas.
¿Consideraciones individuales o colectivas?

Desde 1976, la CIPR promueve el empleo de otro indicador del nivel de protección radiológica: la dosis colectiva. La dosis colectiva consiste esencialmente en la suma de las dosis efectivas que reciben las distintas personas que trabajan con procesos en que se utilizan fuentes de radiación (dosis colectiva ocupacional), o la suma de las dosis efectivas que reciben o recibirán en el futuro los miembros de una población como consecuencia del funcionamiento de una instalación con fuentes de radiación (dosis colectiva del público). La dosis colectiva se expresa en sievert-persona (Svp). Para facilitar su cálculo, se clasifica a la población expuesta en grupos según los niveles de dosis efectiva media y se la define formalmente como se menciona en el cuadro 1.

La dosis colectiva se utiliza como indicador del detrimento colectivo que la exposición a las radiaciones ionizantes puede provocar en un grupo de trabajadores o en la población en general. Su validez está condicionada por las hipótesis de linealidad y de ausencia de umbral aplicables a los efectos estocásticos, por lo que el concepto de dosis colectiva puede emplearse solamente cuando las dosis y las tasas de dosis individuales son bajas. La amplia aceptación que ha ganado este concepto se debe a que permite comparar la eficacia de diferentes estrategias de protección radiológica aplicables a una misma fuente, así como los efectos radiológicos adversos provocados por diferentes fuentes (cuadro 3).

En el proceso de revisión llevado a cabo por la CIPR durante los últimos años se ha cuestionado (9) y revalorizado (10) este concepto. Cabe suponer que en sus próximas recomendaciones la CIPR

CUADRO 3. Dosis efectivas anuales promedio y dosis efectivas colectivas mundiales anuales correspondientes a la exposición natural y a la exposición artificial por diversas causas

\begin{tabular}{lcc}
\hline \multicolumn{1}{c}{ Fuentes de exposición en el mundo } & $\begin{array}{c}\text { Dosis efectiva anual } \\
\text { per cápita (mSv) }\end{array}$ & $\begin{array}{c}\text { Dosis efectiva colectiva } \\
\text { mundial (millones de Svp) }\end{array}$ \\
\hline Radiación natural & 2,4 & 14400 \\
Radiodiagnóstico médico & 0,4 & 2400 \\
Pruebas nucleares en la atmósfera & 0,005 & 30 \\
Accidente de Chernobyl, antigua Unión Soviética & 0,002 & 12 \\
Producción de energía nuclear & 0,0002 & 1,2 \\
\hline
\end{tabular}

Fuente: Datos tomados de la referencia 5. 
mantendrá el concepto de la dosis colectiva, aunque probablemente con algún condicionamiento.

\section{EL EQUILIBRIO ENTRE LOS BENEFICIOS Y LOS RIESGOS: LA REGULACIÓN}

La protección radiológica no debe reducirse a la formulación de buenos propósitos. ¿Cómo lograr que el diseño, la construcción, la operación y el desmantelamiento final de una instalación cumplan con los recaudos técnicos necesarios? La capacitación y el entrenamiento de las personas implicadas es, sin duda, una condición esencial, pero no es suficiente. Es necesario que en cada país funcione una organización independiente para supervisar el cumplimiento de los principios y las normas específicas, es decir, una autoridad reguladora en materia de protección radiológica. Su organización es responsabilidad de los gobiernos y los organismos internacionales especializados pueden brindar asistencia al efecto.

En las Américas, la Organización Panamericana de la Salud (OPS) ha colaborado desde la década de 1960 con los Estados Miembros en la elaboración de normas de protección radiológica y en el desarrollo de actividades de control de las fuentes de radiación (11). En 1997, la OPS publicó un libro con abundante información sobre la organización y el desarrollo de los servicios de imaginología y radioterapia, en el que se presentan los principales conceptos sobre protección radiológica y aspectos reguladores (12).

El Organismo Internacional de Energía Atómica (OIEA), junto con la OPS, la Organización Mundial de la Salud (OMS), la Organización Internacional del Trabajo (OIT) y la Organización de las Naciones Unidas para la Agricultura y la Alimentación (FAO), así como la Agencia Nuclear de Energía (NEA) de la Organización de Cooperación y Desarrollo Económicos, han elaborado varios documentos orientados a apoyar el establecimiento de organizaciones reguladoras nacionales. El primero de ellos, "Normas básicas internacionales para la protección contra las radiaciones ionizantes y la seguridad de fuentes de radiación" (NBIS) (13), que se publicó en inglés en 1996 y en 1997 en castellano, constituye una guía práctica para la estructuración de las normas de protección radiológica y las funciones reguladoras en los países.

El OIEA brinda asistencia a los Estados Miembros que la requieran para la creación y el desarrollo de organizaciones reguladoras de la protección radiológica mediante el llamado Proyecto Modelo. En la actualidad, 13 países latinoamericanos participan en ese proyecto ${ }^{2}$.

Para mayor información se puede consultar la página del OIEA en Internet http://www.iaea.org

\section{Los gobiernos y las funciones reguladoras}

Los gobiernos deben prestar atención a asuntos muy disímiles y administrar los recursos de los países para satisfacer las necesidades básicas de sus pueblos de acuerdo con el grado de desarrollo alcanzado. Cabe preguntarse, ¿en qué medida la atención de los riesgos asociados con la evolución de la tecnología puede ser un objetivo central en países donde las necesidades elementales -como la atención médica o el saneamiento básico- no están satisfechas aún? ¿Qué prioridad puede tener un programa de protección radiológica en tales circunstancias?

Sin embargo, es frecuente encontrar instalaciones médicas con fuentes de radiación ionizante de cierta complejidad en países que tienen grandes carencias tecnológicas. Por lo tanto, el análisis no debe estar dirigido a considerar la prioridad que puede tener un programa de protección radiológica en el contexto del desarrollo general de un país, sino en el marco del desarrollo de un campo específico de aplicación de las fuentes de radiación en ese país. Los costos de protección radiológica y del sistema regulador deben formar parte de los costos de la tecnología que emplea tales fuentes y, desde esa perspectiva, tales costos son relativamente bajos.

No todos los países cuentan con organismos reguladores apropiados. En algunos casos esto puede atribuirse a una subestimación de los riesgos asociados con las fuentes de radiación ionizante. Sin embargo, no es desdeñable la exposición de la población provocada por las fuentes artificiales de radiación, en particular las de uso médico (cuadro 3), a lo que deben agregarse los numerosos accidentes que se producen en el mundo con fuentes industriales y médicas. Los accidentes ponen en evidencia la existencia de fallas extremas en los sistemas de seguridad radiológica, pero cabe suponer que fallas menos graves -que impliquen sobreexposiciones menores- pueden pasar inadvertidas o no notificarse.

\section{Las autoridades reguladoras}

En muchos países, la regulación de la protección radiológica se organizó a medida que se desarrollaban las aplicaciones con fuentes de radiación ionizante y materiales nucleares. En la mayoría de los casos, la organización de la regulación recayó en los organismos de energía atómica o sus equivalentes. Sin embargo, en algunos países los organismos de salud asumieron esa responsabilidad. Durante las últimas décadas aumentó la convicción de que los organismos reguladores debían constituir estructuras gubernamentales diferentes de las instituciones que empleaban fuentes de radiación o que promovían su uso y gradualmente se fueron creando estructuras reguladoras independientes en varios paí- 
ses. Es facultad de cada país decidir la organización más apropiada para sus condiciones específicas.

Las autoridades reguladoras deben establecer normas de protección radiológica y verificar su cumplimiento durante todas las etapas de desarrollo de las diferentes prácticas que empleen fuentes de radiación ionizante. Estas autoridades deben tener la suficiente capacidad de acción técnica, legal y ética que les permita ejercer su autoridad sobre las personas y entidades encargadas de tales prácticas. El principal mecanismo con que cuentan las autoridades reguladoras para aplicar su autoridad consiste en un sistema de licencias institucionales que autorizan tener, utilizar, transferir y trasladar fuentes de radiación ionizante o realizar cualquier operación con ellas. Estas autorizaciones o licencias institucionales están condicionadas al cumplimiento de determinados requisitos de diseño y operación de las instalaciones, equipos y fuentes. Además, es preciso contar con un sistema de autorizaciones o licencias personales que se otorgan al personal según su nivel de capacitación y entrenamiento en protección radiológica y en la práctica específica en que utilizarán las fuentes de radiación.

Las autoridades reguladoras no se pueden limitar a cumplir funciones administrativas, sino que deben estar en condiciones de evaluar la protección y la seguridad radiológicas en el ámbito de cada fuente y en las condiciones particulares de cada tipo de práctica, y de exigir las mejoras que resulten necesarias. Esto solo es posible si se cuenta con personal profesional y técnico altamente capacitado, con experiencia en la protección radiológica y - lo que no es menos importante- experiencia en la operación del tipo de instalaciones que se supervisa.

Se debe recordar que en condiciones normales, las dosis de radiación que reciben las personas no provocan alarma sensorial ni manifestaciones clínicas inmediatas, aunque ello no significa que los riesgos sean nulos ni pequeños. Por consiguiente, la autoridad reguladora debe exigir que cada instalación cuente con sistemas de vigilancia radiológica que permitan evaluar las dosis de radiación recibidas por las personas.

Desde la publicación de las NBIS (13), el OIEA, junto con los demás organismos responsables de esa publicación, ha promovido el fortalecimiento de estructuras reguladoras nacionales y ha brindando asistencia técnica al respecto. La Conferencia Internacional sobre Infraestructuras Nacionales Reguladoras en Seguridad Radiológica, celebrada en Rabat, Marruecos, en septiembre de 2003, constituye una prueba de la importancia que se le otorga a la regulación (14).

\section{LA FUNCIÓN DE LAS AUTORIDADES DE SALUD}

Las funciones de las autoridades nacionales de salud en el proceso de regulación difieren según el país. Sin embargo, las autoridades sanitarias tienen la responsabilidad de emitir opiniones autorizadas en materia de salud, así como las autoridades reguladoras en protección radiológica tienen la misión de preservar la salud de las personas ante este riesgo específico. Por ello, independientemente de cómo se estructuren las funciones reguladoras en un país, las autoridades sanitarias no deberían estar desvinculadas del sistema regulador. Su opinión especializada puede contribuir de manera importante a validarlo.

Las autoridades de salud pueden participar activamente al menos en tres aspectos de la protección radiológica: la promoción de la educación formal del personal de salud en lo concerniente a la protección radiológica, la atención médica de las personas sobreexpuestas y la protección radiológica de los pacientes.

\section{Formación del personal de salud}

La formación universitaria y los procesos formales de entrenamiento para los profesionales de la salud deben aportar los conocimientos necesarios sobre el empleo de las radiaciones ionizantes en las aplicaciones médicas, sus riesgos y beneficios. En el caso de los especialistas, esto se debe complementar con una profunda capacitación y un intenso entrenamiento en las aplicaciones médicas específicas. Los físicos médicos son indispensables en los servicios de radioterapia, conviene su presencia en los de medicina nuclear y deberían asesorar en los de radiodiagnóstico. Aunque esta no es una especialidad nueva, en algunos países no se cuenta con un número suficiente de físicos médicos ni con instituciones apropiadas para su formación. Las autoridades de salud pueden contribuir de un modo importante al desarrollo, la consolidación y el reconocimiento de esta especialidad.

\section{La atención médica de las personas sobreexpuestas a radiaciones}

Otro aspecto en el que las autoridades de salud deberían desempeñar un papel protagónico es la atención médica de las personas sobreexpuestas accidentalmente. La evaluación diagnóstica, la estrategia terapéutica que debe seguirse y las derivaciones apropiadas incumben directamente a los profesionales de la salud y deben planificarse estableciendo los acuerdos correspondientes con las instituciones médicas involucradas.

\section{La protección radiológica de los pacientes}

Las fuentes de radiación ionizante de uso médico son las más numerosas y las que contribuyen 
en mayor medida a la exposición artificial de la población (cuadro 3). En la actualidad hay más de un millón y medio de fuentes de radiación ionizante declaradas en el mundo, tanto para diagnóstico como para tratamiento médico, y con ellas se realizan más de dos mil millones de procedimientos anualmente (cuadro 4). Además de provocar la inevitable exposición de los trabajadores y de algunos miembros de la población - como todas las fuentes de radiación ionizante-, estas fuentes están concebidas para irradiar deliberadamente a determinadas personas: los pacientes. La CIPR ha dedicado una docena de publicaciones al tema de la protección de los pacientes y en una de ellas (15) ha sintetizado los criterios apropiados para las prácticas de diagnóstico y tratamiento.

La protección radiológica del paciente está relacionada con el ejercicio de la profesión médica. En ocasiones, los organismos reguladores se limitan a sí mismos en aras de no "invadir" el campo médico (16) y se produce entonces un vacío que ningún organismo cubre. Por ello es deseable que las autoridades de salud se interesen especialmente en la protección radiológica del paciente y establezcan una acción coordinada con los organismos reguladores.

\section{¿Cómo proteger a personas irradiadas deliberadamente?}

Para obtener una imagen radiológica o tratar un tumor es indispensable que el paciente reciba cierta dosis de radiación. Puede entonces hacerse referencia a una dosis necesaria. Sin embargo, en ocasiones la exposición es mayor que la requerida para

CUADRO 4. Principales equipos médicos que emplean radiaciones ionizantes y número de procedimientos realizados anualmente en el mundo

\begin{tabular}{lr}
\hline \multicolumn{1}{c}{ Aplicación médica } & Número de equipos \\
\hline Radiodiagnóstico (1910 millones & \\
de procedimientos por año) & 700000 \\
$\quad$ Convencional & 34000 \\
$\quad$ Tomografía computarizada & 40000 \\
$\quad$ Mamografía & \\
Radiodiagnóstico dental (520 millones & \\
de procedimientos por año) & 900000 \\
$\quad$ Convencional & \\
Medicina nuclear (32 millones & 14000 \\
de procedimientos por año) & 300 \\
$\quad$ Escáner rectilíneo, cámara gamma & \\
$\quad$ Tomografía por emisión de positrones & \\
Radioterapia & \\
(4,7 millones de tratamientos por año) & 5000 \\
$\quad$ Rayos X & 4000 \\
$\quad$ Cobalto & 5000 \\
$\quad$ Acelerador lineal &
\end{tabular}

Fuente: Datos tomados de la referencia 5. el procedimiento o se exponen tejidos que están fuera del campo de interés, lo que genera una dosis innecesaria. No se pueden establecer límites de dosis para los pacientes, ya que la relación dosis-beneficio es diferente en cada caso. Los conceptos de justificación y optimización adquieren entonces mayor importancia: el médico debe analizar la justificación de cada procedimiento que prescribe y el equipo médico especializado que lo realiza debe optimizarlo (16).

La optimización está estrechamente relacionada con la calidad de los procedimientos. En algunas instituciones las dosis son hasta 10 veces mayores que las empleadas en otras para realizar estudios radiodiagnósticos equivalentes desde el punto de vista de la información obtenida. Esto significa que la dosis innecesaria puede ser muy elevada en algunos casos. Se debe tener en cuenta que la dosis colectiva mundial generada por los procedimientos de radiodiagnóstico médico es la mayor de las atribuibles a la exposición artificial a las radiaciones ionizantes (cuadro 3). Se puede suponer que si se lograra mejorar la calidad de los procedimientos radiológicos se podría reducir la correspondiente dosis colectiva mundial al menos a la mitad sin menoscabar los beneficios del radiodiagnóstico.

Las dosis efectivas individuales en los procedimientos de radiodiagnóstico pueden variar, según el tipo de estudio, desde decenas de $\mu \mathrm{Sv}$ hasta más de $100 \mathrm{mSv}$. Algunas técnicas pueden originar dosis absorbidas de 10 a 100 mGy en algunos tejidos, como la tomografía computarizada (17), o de 1 Gy o más en la piel, como la radiología intervencionista (18). En ocasiones, la dosis absorbida puede sobrepasar los umbrales de los efectos deterministas y producir lesiones en los pacientes.

En radioterapia, las dosis prescritas para el tratamiento de los tumores son elevadas (entre $20 \mathrm{y}$ 85 Gy). La eficacia de estos procedimientos depende en gran medida de la exactitud con que se irradia al paciente y del grado en que se logra minimizar la exposición de los tejidos sanos, es decir, depende tanto del valor de la dosis como de la localización del campo de irradiación. Dosis superiores a la prescrita aumentan el riesgo de lesiones y muerte provocadas por las radiaciones, mientras que dosis inferiores aumentan el riesgo de un tratamiento ineficaz. La desviación aceptable para minimizar estos efectos indeseables es de $5 \%$.

\section{Accidentes con fuentes médicas}

En un estudio realizado por el OIEA en el que se investigaron 90 accidentes relacionados con la radioterapia en diversos países, los errores detectados de sobreexposición son tres veces más numerosos que los de subexposición (19). Esta asimetría podría relacionarse en parte con el hecho de que la detec- 
ción de sobreexposiciones se ve favorecida por reacciones clínicamente observables en el paciente, en tanto que la subexposición no produce manifestaciones clínicas, aunque puede dar lugar a un tratamiento menos eficaz y a la posible muerte del paciente por la evolución de su enfermedad. Esas consecuencias, aunque graves, pueden no ser tan patentemente atribuibles a subexposiciones provocadas por errores en los procedimientos.

Durante la última década, en las Américas se han producido dos accidentes con fuentes de radioterapia que han provocado la muerte de decenas de pacientes por sobreexposición $(20,21)$. Graves fallas condujeron a esos accidentes. Cabe preguntarse, ¿cuántos casos sin detectar o sin notificar pueden haber ocurrido en el mundo, con desviaciones menos patentes en los valores de las dosis, pero con un detrimento significativo de la eficacia de los tratamientos?

El empleo de un medio terapéutico como la radiación se justifica si el procedimiento se planifica con la mejor técnica disponible y se aplica con la mayor calidad (22). La prevención de accidentes en la práctica de la radioterapia vincula estrechamente dos especialidades: la protección radiológica y la física médica (23).

\section{El plan de acción internacional para la protección radiológica de los pacientes}

En marzo de 2001 se celebró en Málaga, España, la Conferencia Internacional sobre Protección Radiológica del Paciente, patrocinada por varias organizaciones internacionales (OIEA, OMS, OPS, entre otras). Como resultado de esa conferencia se aprobó un plan de acción internacional para la protección radiológica de los pacientes (24) que contempla, entre otras, las acciones siguientes:

- promover la educación y el entrenamiento de médicos clínicos, especialistas, tecnólogos, enfermeros, físicos médicos, radiofarmaceutas, diseñadores de equipos, ingenieros de mantenimiento, administradores, entre otros, en la protección radiológica, el control de la calidad y la reducción de las dosis innecesarias y de los riesgos de exposición accidental en las aplicaciones médicas

- promover el intercambio de información sobre esos temas entre instituciones de diversos países

- promover el reconocimiento de la importancia de los tecnólogos en la protección radiológica de los pacientes y mejorar su capacitación

- promover el reconocimiento de la importancia de los físicos médicos como profesionales de la salud

- preparar guías apropiadas destinadas al personal de los servicios médicos que emplean fuentes de radiación
- reconocer que existen aspectos relacionados con la transferencia de equipos de segunda mano a países en desarrollo que inciden en la protección radiológica y proporcionar guías destinadas a los donantes de equipos de segunda mano, los receptores y las organizaciones intermediarias. En particular se debe tomar en cuenta la necesidad real del país receptor, la provisión de herramientas, accesorios; piezas de recambio y manuales; la formalización de acuerdos para las pruebas de aceptación, instalación y mantenimiento, y el entrenamiento en el uso de equipos con sus dispositivos específicos de protección.

Se espera que las autoridades de salud conozcan este documento y participen activamente en la puesta en práctica de estas acciones.

\section{CONCLUSIONES}

Lejos estaban Roentgen y Becquerel de imaginar la extraordinaria expansión de las aplicaciones prácticas de sus descubrimientos. Pero en la actualidad no se desconocen, como en aquellos tiempos, los riesgos asociados con esos fenómenos y no se justifica que las prácticas con fuentes de radiación ionizante, que tantos beneficios pueden aportar, provoquen daños por la falta de medidas eficaces de protección radiológica y de procedimientos adecuados de garantía de la calidad.

Ese equilibrio entre beneficios y riesgos se puede controlar mediante sistemas reguladores nacionales adecuados. Es responsabilidad de los gobiernos crear condiciones jurídicas y administrativas apropiadas para que las autoridades reguladoras de la protección radiológica lleven a cabo su labor eficazmente.

Las autoridades de salud, aun cuando no ejerzan funciones reguladoras directas en esta materia, pueden contribuir de un modo importante mediante su acción coordinada con los órganos reguladores para proteger la salud de los trabajadores y miembros de la población contra los riesgos que implican las fuentes de radiaciones ionizantes. También deberían cumplir una importante función en la organización de los medios necesarios para la atención de personas sobreexpuestas por accidentes con fuentes de radiación. Pero cabe esperar que las autoridades sanitarias desempeñen un papel protagónico en la protección de los pacientes debido a su relación directa con la profesión médica. Para ello, deben contar con profesionales sanitarios con conocimientos en materia de protección radiológica, promover el uso del equipo adecuado y aplicar procedimientos apropiados de garantía de la calidad.

La intervención oportuna de las autoridades nacionales de salud puede contribuir en gran me- 
dida a reducir las dosis innecesarias en las prácticas médicas con fuentes de radiación y reducir las probabilidades de que ocurran accidentes radiológicos en este campo.

\section{SYNOPSIS}

\section{Regulating radiological protection and the role of health authorities}

This article summarizes the development of protection against ionizing radiation and explains current thinking in the field. It also looks at the decisive role that regulatory agencies for radiological protection must play and the important contribu- tions that can be made by health authorities. The latter should take an active part in at least three aspects: the formal education of health personnel regarding radiological protection; the medical care of individuals who are accidentally overexposed, and the radiological protection of patients undergoing radiological procedures. To this end, health professionals must possess sufficient knowledge about radiological protection, promote the use of proper equipment, and apply the necessary quality assurance procedures. Through their effective intervention, national health authorities can greatly contribute to reducing unnecessary doses of radiation during medical procedures involving radiation sources and decrease the chances that radiological accidents will take place.

Key words: radiation, ionizing; radiation protection; radiation control; standards.

\section{REFERENCIAS}

1. International Commission on Radiological Protection. The 1990 recommendations of the International Commission on Radiological Protection. Ann ICRP. 1991;21:1-3. (ICRP Publication No. 60).

2. Taylor L. Radiation protection standards. London: CRC Press; 1971.

3. Skvarka J, Aguirre A. Normas y estándares aplicables a los campos electromagnéticos de radiofrecuencias en América Latina: guía para los límites de exposición y los protocolos de medición. Rev Panam Salud Publica. 2006;20(2/3):205-12.

4. International Commission on Radiation Units and Measurements. Quantities and units in radiation protection dosimetry. Oxford: ICRU; 1993. (ICRU Report No. 51).

5. Comité Científico de las Naciones Unidas para el Estudio de los Efectos de las Radiaciones Atómicas. Informe 2000. Viena: UNSCEAR; 2000.

6. Comité Científico de las Naciones Unidas para el Estudio de los Efectos de las Radiaciones Atómicas. Informe sobre efectos hereditarios. Viena: UNSCEAR; 2001

7. International Commission on Radiological Protection. Protection from potential exposure: a conceptual framework. Ann ICRP. 1993;23:1. (ICRP Publication No. 64).

8. International Commission on Radiological Protection. Protection from potential exposures: application to selected sources. Ann ICRP. 1997;27:2. (ICRP Publication No. 76).

9. Clarke R. The evolution of the system of radiological protection: the justification for new ICRP recommendations. J Radiol Prot. 2003;23:129-42.
10. Arias C. ICRP versus ICRP: challenges for future ICRP recommendations. Proceedings of the XI International Congress of Radiation Protection, Madrid, May 2004. Madrid: Sociedad Española de Protección Radiológica; 2004.

11. Hanson G, Borrás C, Jiménez P. History of the radiological health program of the Pan American Health Organization. Rev Panam Salud Publica. 2006;20(2/3): 87-98.

12. Organización Panamericana de la Salud. Organización, desarrollo, garantía de calidad y radioprotección en los servicios de radiología: imaginología y radioterapia. Washington, D.C.: OPS; 1997.

13. International Atomic Energy Agency. Normas básicas internacionales para la protección contra las radiaciones ionizantes y la seguridad de las fuentes de radiación. Viena: OIEA; 1996. (Colección Seguridad No 115).

14. International Atomic Energy Agency. Findings and recommendations of the International Conference on National Infrastructures for Radiation Safety; 2003 Sept; Rabat, Morocco. Vienna: IAEA; 2004

15. International Commission on Radiological Protection. Radiological protection and safety in medicine. Ann ICRP. 1996; 26(2):1-47. (ICRP Publication No. 73).

16. Arias C. Regulatory functions and medical exposure. Proceedings of IX International Congress of Radiation Protection; 1996 Apr; Vienna, Austria. Vienna: Austrian Association for Radiation Protection; 1996.

17. International Commission on Radiological Protection. Managing patient dose in computed tomography. Ann ICRP. 2000; 30(4):1-45. (ICRP Publication No. 87).

18. International Commission on Radiological Protection. Avoidance of radiation injuries from medical interventional procedures. Ann ICRP. 2001;30(2):1-67. (ICRP Publication No. 85).

19. International Atomic Energy Agency. Lessons learned from accidental exposures in radiotherapy. Vienna: IAEA; 2000. (Safety Report Series No. 17)

20. International Atomic Energy Agency. Accidental overexposure of radiotherapy patients in San José, Costa Rica. Vienna: IAEA; 1998.

21. International Atomic Energy Agency. Investigation of an accidental exposure of radiotherapy patients in Panama. Vienna: IAEA; 2003

22. International Commission on Radiological Protection. Prevention of accidents to patients undergoing radiation therapy. Ann ICRP. 2001;30(3):1-70. (ICRP Publication No. 86).

23. Arias C, Piumetti E, Sanchez G. Medical sources and radiological accidents. Proceedings of the World Congress on Medical Physics and Biomedical Engineering, 1994 Aug; Rio de Janeiro, Brazil. Rio de Janeiro: Brazilian Society of Medical Physics; 1994.

24. International Atomic Energy Agency. International action plan for the radiological protection of patients. Vienna: IAEA; 2002.

Manuscrito recibido el 14 de diciembre de 2004 Aceptado para publicación, tras revisión, el 30 de agosto de 2005 . 